\title{
PRINCIPALES DETERMINANTES DE LOS PRECIOS DE LA TIERRA EN LA ZONA PAMPEANA, 1950-1985
}

\section{INTRODUCCION}

El análisis de la evolución de los valores de la tierra es un tema de importancia en el ámbito de la economia agraria argentina, de manera especial por sus implicancias en materia de politica tiscal, crediticia y de formación de los precios en los mercados agricolas. Pero apenas intentamos abordar el tema, nos encontramos con el problema de definir que se entiende por "tierra", pues si se trata de las cualidades intrinsecas que esta posee, sólo debemos tener en cuenta en la valorización estos componentes, separando cuidadosamente el capital incorporado, incluso en forma de fertilizantes y agroquimicos asi como tambien todo agente que haya alterado estas cualidades esenciales, tales como los procesos de degradación de los suelos.

Sin embargo, si nos volvemos demasiado rigurosos en estas apreciaciones, las posibilidades de estudio de los precios de la tierra serian practicamente nulas, por lo cual trataremos de alcanzar un nivel de precisión lo más aceptable posible, dejando de lado en la selécción aquellos establecimientos agropecuarios que presentan mejoras excepcionales, que estuviesen demasiado cerca de los centros urbanos y todo otro aspecto inusual que se considere perturbador a los efectos del presente trabajo.

Preocupa desde el inicio dejar claras estas cuestiones que,aunque parecieran formales, son centrales a los fines de: a) trazar la evolución de los precios de la tierra en la zona sur pampeana entre 1950-1985; b) determinar los factores de mayor importancia que han inferido en la formación de tales precios; y c )cuantificar, en la medida de lo posible, la influencia que han ejercido las variables identificadas en el lapso estudiado.

El trabajo comprende cuatro partes. En la primera se hace una breve referencia a la teoria económica de la renta de la tierra a fin de arribar a la concepción ac- 
tual del precio de la tierra como resultante del flujo actualizado de dicha renta. En la segunda parte se analizan los factores que afectan el valor de la tierra a traves de su tratamiento en un conjunto de trabajos seleccionados y publicados en Argentina y en otros países. En la tercera parte, se consideran los factores que se entiende son más relevantes en la formación del precio de la tierra en Argentina, tanto a los por asi llamarlos "tradicionales" como a los que han pasado a cobrar peso en las ultimas decadas. Por ultimo, en la cuarta parte, se intentan en base a los datos recogidos y la definición de un área geográfica lo más homogenea posible algunos ensayos de medicion para estimar el efecto que produce cada una de las variables identificadas sobre los precios de la tierra.

\section{RENTA DE LA TIERRA}

Se trata de dilucidar dos cuestiones preliminares: a) que se entiende por renta de la tierra? y b) la renta es consecuencia o efecto de los precios o, por el contrario, es causa de los precios de los productos agrícolas?

Sobre el primer aspecto planteado, Ricardo nos da una cuidadosa definición: "la renta de la tierra es aquella parte del producto que se paga al terrateniente por el uso de las energias originales e indestructibles del suelo" (Ricardo, 1959, p. 51). En cuanto al segundo aspecto, las posiciones de Ricardo y Smith,quienes, entre los precursores, procuraron dilucidar el tema, son encontradas. Es tal vez Ricardo, quien lo define en forma generalmente más aceptada como consecuencia del precio y no como una de sus causas. Refiriendose a un cereal específico, escribe: "Dicho cereal (el trigo) no se encarece porque hay que pagar una renta, sino que debe pagarse una renta porque el cereal es caro; y como se acaba de observar, no acaecería reducción alguna en el precio del cereal aunque los terratenientes condonasen la totalidad de sus rentas" (Ricardo, 1959 p. 56).H. von Thunen en un intento de formular una teoria de la localización agrícola considera como factor determinante la renta económica por unidad de superficie, la que a su vez es función de la distancia con respecto al mercado, con lo cual la renta de la tierra queda expresada como:

$$
R=E(p-a)-E\left(f^{r}\right)
$$


donde $R=$ renta por unidad de superficie; $E=$ rendimiento natural por ha.; $p=$ precio de mercado por quintal; $a$ = gasto por ha.; $f$ = flete y $r$ = distancia al mercado. Este factor locacional determina ventajas para las tierras mejor ubicadas con respecto a los mercados de los productos agrícolas, pues a mayor distancia los fletes irán absorbiendo la renta económica agrícola y disminuirán el interes por la compra de dichas tierras. La influencia de los costos de transporte, sin embargo, parece haber perdido fuerza en los países industrializados, y en la actualidad, sólo tiene vigencia relativa en los países en desarrollo.

Man distingue dos conceptos de renta, la renta absoluta como: "la plusvalía producida en la agricultura por encima de la ganacia media" (Marx, 1959, p. 708), para lo cual debe darse una composición orgánica del capital agrario inferior a la del no agrario, y a la renta diferencial de tipo I en la que incluye la posibilidadde disparidades de renta originadas en diferencias de fertilidad y de situación de las diferentes clases de tierras, concepto este ultimo de utilidad empírica actual.

El análisis neoclásico acepta la posición ricardiana. Marshall (1907) introduce el concepto de cuasi-renta, determinada tambien por los precios tanto para la sociedad en su conjunto como para el empresario individual; y siguiendo el tratamiento neoclásico tradicional, la tierra es remunerada de acuerdo a su contribución en el proceso productivo, es decir, la productividad marginal que a fade.

En una concepción más moderna, se apela a la fórmula de actualización tan usada en matemática financiera con el fin de expresar el precio de la tierra (que es un valor de stock) en terminos de una variable flujo como es la renta o rentabilidad del servicio productivo tierra en el proceso agropecuario.

$$
P_{t}=\frac{R_{1}}{(1+i)}+\frac{R_{2}}{(1+i)^{2}}+\frac{R_{3}}{(1+i)^{3}}+\ldots \ldots+\frac{R_{n}}{(1+i)^{n}}
$$

donde $\mathrm{R}=$ renta; $\mathrm{Pt}=$ precio de la tierra en el momento $\mathrm{t} ; \mathrm{e} \mathrm{i}=$ tasa de descuento elegida. En el caso de la tierra, la fórmula se simplifica a partir de los supuestos como $n=\infty, i>0$ y $R 1=R 2=R 3=\ldots=$ Rn de la manera siguiente: 


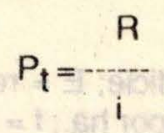

En el análisis moderno, se suele asumir que el empresario se comporta como "un diversificador de cartera" o sea, el individuo compara la rentabilidad proveniente de su inversión en tierras agricolas con las ganancias provenientes de otras alternativas de inversión en tierras agrícolas y a la vez examina el riesgo asociado a dichas actividades.

Finalmente, T. Schultz (1953) plantea la declinación de la importancia económica de la tierra en tanto esta se va convirtiendo en una proporción menor de los insumos usados en la agricultura y ha dejado, por lo tanto, de ser un factor limitativo que imponia serias restricciones al incremento en la producción. Si esto es verdad, y los componentes del capital no directamente relacionados con la productividad del suelo han tenido una tendencia creciente en los ultimos af́os, la renta pura, producto de las propiedades originales y naturales de la tierra, debiera influir cada vez menos en los precios de la tierra.

\section{FACTORES QUE AFECTAN EL VALOR DELA TIERRA: SU TRATAMIENTO EN UN CONJUNTO SELECCIONADO DE ESTUDIOS}

La variable que aparece en todos los trabajos que se han ocupado de este tema y que hemos consultado para abordar este estudio es, por su importancia y su relación directa con el precio de la tierra, el ingreso derivado de la explotación agropecuaria.

Una parte considerable del valor venal de la tierra se suele asignar a su potencial económico productivo. Por lo tanto, se considera util medir los precios de la tierra en la cantidad del producto principal que puede obtenerse de ella y que se necesite para comprar una hectarea. Los trabajos empíricos realizados hasta el momento se apoyan en el ingreso neto, o bien, en aquellos casos que no se dispusiere de series de costos agropecuarios, en los ingresos brutos. Hoover (1960) prueba mediante un modelo de expectativas basadas sobre el ingreso neto esperado (al estilo del modelo de Nerlove) que para el periodo 1911-1958 por él estudiado en E.E.U.U., los cambios de la renta año a año no producían másque un impacto 
pequeno sobre el valor de la tierra. En cambio, la variable considerada, ingreso neto del campo, tomada con un retardo de tiempo de un afio, resulta ser altamente significativa en los trabajos de Heady y Tweeten (1963) y Van Vuuren (1968).

El cambio tecnológico ha merecido una atención especial en los trabajos de autores argentinos. Si bien es dable captarlo a traves de los ingresos brutos formando el componente producción física o rendimientos por hectárea, era necesario fijar posición sobre la neutralidad o falta de neutralidad de tal variable frente a los precios de la tierra. A tal fin, se subdividió el período de desarrollo del sector agropecuario en distintos subperíodos. Hasta1939, el proceso de desarrollo se caracterizó por la expansión de la frontera agrícola sin cambios importantes en el uso de los factores de la producción que, por lo tanto, no incidieron en el precio de la tierra. Para el periodo de 1940-1960, se adoptó un modelo asentado principalmente en una estructura combinada agrícola-ganadera diversificada a fin de adaptarse a las variaciones de precios del mercado internacional y disminuir los riesgos. En este lapso de tiempo, el factor de mayor flexibilidad fue la tierra; de alli que su precio se econtrara deprimido frente al costo de oportunidad del capital.

En las ultimas dos decadas, se produce una marcada incorporación de tecnología, pero sus rasgos difieren segun el periodo que se considere. En la decada del 60, se adoptan innovaciones de tipo mecánico "ahorradoras de mano de obra", pero neutrales en cuanto a los rendimientos por hectárea, por lo que aparentemente no pudieron haber afectado los valores de la tierra.

La década del 70, en cambio, incorpora paquetes tecnológicos destinados esencialmente a aumentar la productividad de la tierra.Son claramente "ahorradores de la tierra", con lo cual los aumentos de rentabilidad parecerian indicar que esta vez la tecnología no permaneció neutral en la formación del valor de la tierra. Aun más, estos paquetes tecnológicos han originado un nuevo esquema productivo. De la forma tradicional de explotaciones mixtas de la región pampeana, los productores se ha volcado hacia el uso intensivo del factor tierra en actividades netamente agricolas. Como resultado, las tierras agrícolas se cotizan a precios superiores que las sólo aptas para ganadería. Sin embargo,en los ultimos años, 19801984, la sobreoferta de granos con la consiguiente caída de los precios ha revertido la situación característica de la decada del 70. Los excedentes provenientes del cambio tecnológico tienden a desaparecer, con lo que los precios de la tierra vuelven paulatinamente a ser neutrales al impacto tecnológico. El análisis de la repercusión de los impuestos se centró enel impuesto a la tierra y sus formas alternativas de aplicación, es decir, las características ecológicas de la tierra o la productividad media actual de regiones homogeneas. En general, autores como Martín Pineiro (1970), E. C. Passour (1973), coinciden en afirmar que los incrementos de las tasas impositivas no pueden trasladarse a los precios de los productos agricolas para ele- 
varlos, pero se reflejan en una disminución en la renta de la tierra y, por lo tanto, en su precio de mercado.

En el ámbito de la política agropecuaria se han tomado otros conjuntos de medidas que directa o indirectamente han tenido gran repercusión en la formación del precio de la tierra. Nos referimos sobre todo a los programas de precio sosten. Duane G. Harris (1977) señala al respecto, que toda ganancia garantizada a los productores que va más allá de los gastos de explotación es capitalizada en valores más altos de la tierra. En cambio, no se encuentran mencionados en los trabajos consultados otros instrumentos de política económica, tales como creditos subsidiados, tipos de cambio preferenciales, exenciones impositivas, etc. Una excepción la constituye Sojit cuando expresa: "Indudablemente un incremento en la cantidad de credito disponible en el mercado hipotecario tendrá una repercusión inmediata en el mercado de tierra. Pero los aumentos generales en la liquidez del publico liberarán a los recursos financieros los que si la tierra es un "bien preferido" aumentarán su demanda"(Sojit, 1968, pág. 16).

El sistema de arrendamientos, propio de casi todos los paises latinoamericanos, ha sido manejado bastante defectuosamente al punto de producir a lo largo de dos decadas, un clima de inseguridad jurídica que provocó, al menos en Argentina, graves distorsiones en los mercados de tierras. Esta variable es difícil de cuantificar, pero no debe dejar de mencionarse el importante rol que ha jugado en el desestímulo a la oferta de tierras agrícolas. Hay dos formas de elevarla demanda de tierra, una como consecuencia del crecimiento de la población que, al requerir mayor cantidad de alimentos y fibras, presiona sobre el mercado de tierras elevando los precios de las mismas; y la segunda, a raiz de rápidos procesos de industrialización que, al producir un desarrollo urbano creciente, las tierras rurales compiten como rivales de las urbanas, encareciendose el precio de las primeras. El problema que se plantea en este caso es econtrar la variable que refleje esta presión de la población. V. Ruttan (1961), E Schuh y Scharlach W. (1963) usan la densidad de población entre períodos censales y la consideran altamente significativa al explicar los diferenciales valor-tierra en el corte transversal.

Finalmente, la inflación ha motivado la necesidad de convertir el dinero, cuya función de "reserva de valor" se pierde a medida que el nivel de precios sube, en otros activos entre los que se encuentran los bienes duraderos y entre ellos, de manera especial "la tierra"; esta ultima se vuelve asi una alternativa de inversión atrayente para aquellos sectores rurales y no rurales que buscan un resguardo frente a la inflación. Este hecho, sefialado entre otros por Wantrup y Ciriacy (1964) en E.E.U.U.,Saturnino Zemboraín (1973) y Norberto Ras y R. Levis (1977) en Argentina, ha modificado el comportamiento en el mercado de tierras, y originado en varias 
oportunidades alzas espectaculares en los precios de las tierras no acompanadas por aumentos en los ingresos del sector agropecuario.

\section{LOS FACTORES MAS RELEVANTES DELOS PRECIOS DELA TIERRA EN ARGENTINA}

Los factores senalados en la parte precedente del trabajo parecen apropiados para la temática en consideración.

En primer lugar, se hace referencia a la relación existente entre los precios de la tierra y los del producto o productos principales. En Argentina pareciera que se ha ampliado la brecha existente entre estas dos clases de precios, ya que, mientras los primeros crecieron en las dos ultimas decadas, los segundos han perdido paulatinamente su posición relativa.

Unido a la problemática de los precios de la producción estan insertos aspectos de índole política que hacen a la existencia o no de trabas en la formación de precios en los mercados. Toda vez que el Estado interviene fijando precios sosten, vedas al consumo de carne vacuna, devaluaciones o revaluaciones en el tipo de cambio o toma medidas análogas en el campo de la política económica, se generan implicancias sobre el valor de la tierra a traves de los precios agricolas.

Otro aspecto igualmente vinculado con lo anterior, está dado por los aumentos en los rendimientos físicos de la producción. Muchas veces los aumentos en los ingresos provenientes de la actividad agropecuaria se originan no tanto en mejores retribuciones al producto, sino en incrementos en los coeficientes de productividad por hectárea. De allí la necesidad del tratamiento específico de la varible cambio tecnológico, sobre todo teniendo en cuenta que, si bien la Argentina se encuentra rezagada en la incorporación de nuevas tecnologias en las dos ultimas decadas al igual que el resto de países en vías de desarrollo, ha ido aumentando su participación en el uso de nuevos metodos productivos. Considerando que una de las causas más importantes en la variabilidad de la adopción de tecnologías es el problema de la relación precio del producto/precio del insumo, es conveniente analizar asimismo el costo de los insumos estrategicos como determinante del precio de la tierra a traves de su repercusión en la rentabilidad neta obtenible de la actividad agropecuaria. 
La estructura impositiva en Argentina ha tenido un rol significativo. Los atrasos en la actualización de la base imponible (valor fiscal de la tierra) han producido, en un pais con fuerte proceso inflacionario, una disminución notable en el costo impositivo que, junto a los períodos de desgravaciones impositivas, seguramente han jugado como factores coadyuvantes en las decisiones respecto a la compra de tierra por sectores inversores ajenos al sector rural, que buscan este tipo de alternativas de capitalización.

Por otra parte, el credito destinado al sector agrario a bajas tasas de interés en algunos años ha constituido un verdadero subsidio que de algun modo incentivó asimismo la demanda de tierras por los inversores anteriormente citados.Sería interesante tambien analizar la evolución de la estructura de tenencia de la tierra, pues, de acuerdo al grado de movilidad verificado, podria pensarse que si el factor tierra está sujeto a baja movilidad, los tenedores de tierras, en el marco de un proceso inflacionario, se aferrarian a ella como reserva de valor, y si a su vez se experimentara la entrada de demandantes de fuera del sector, estas dos circunstancias son susceptibles de generar un alza desmedida de precios en algunos períodos. El rápido proceso de industrialización en Argentina inciado en el periodo interbelico produce migraciones internas que originan un proceso de urbanización y una modificación considerable del perfil demográfico. Se experimentan cambios en la densidad de población rural y fuertes presiones en los centros poblados a fin de expandir el conurbano ganando tierras antes dedicadas a fines agropecuarios. Pareciera ser entonces que esta presión hubiera producido cambios en los precios de las tierras rurales cercanas a los centros urbanos; entre otras razones aparte de la seffalado, por factores recreativos y de comodidad. Hasta aqui, en cuanto a los factores por asi llamarlos tradicionales. Pero la tierra como un activo real más, produce una segunda clase de retornos, que son las ganancias de capital provenientes de cambios en su valor real de mercado. En este segundo aspecto, debemos considerar variables de tipo "especulativo" en la demanda de tierras, y en este sentido, junto a la evolución de la tasa de inflación, no debería descuidarse el cambio en la rentabilidad de distintos activos, tales como el dólar, el oro en los mercados internacionales y las cotizaciones de acciones y títulos publicos. No obstante, es probable que en Argentina la tierra como "reserva de valor" sea el activo real que cumple más acabadamente este rol, dada laimperfección en el mercado de capitales.

Asociada al proceso de ganancias de capital se halla la noción de riesgo que cabe considerarla inserta dentro de las expectativas de beneficios originados por la variabilidad en el valor de los activos reales. Si a las ganancias de capital pro- 
venientes de cambios en el valor real de la tierra le adicionamos el menor riesgo que tiene la posesión de este activo comparado con el resto, el prestigio social que implica ser poseedor de tierras y el poder político que va unido en toda America Latina a los detentores de esta clase de activos, resulta que la tasa de apreciación o valorización puede estar por encima de la del conjunto de activos reales.

Finalmente,existe una variable que ha sido descuidada en los trabajos de investigación en nuestro país: la degradación de los suelos, ya sea por factores naturales o mal manejo de los mismos.

Este factor puede afectar en un futuro mediato, de no realizarse prácticas de conservación necesarias, la productividad de la tierra y ocasionar una fuerte baja en la rentabilidad y, por ende, en los precios de la tierra.

\section{PRECIOS DE LA TIERRA EN LA ZONA SUR DE LAREGION PAMPEANA}

\section{Delimitación del área estudiada}

Tiene especial importancia para el presente trabajo trazar los límites de las áreas que componen la Provincia de Buenos Aires de acuerdo con sus aptitudes, tipos de suelo y regimen de precipitaciones. Los precios de la tierra se establecen,obviamente, en función de su aptitud potencial y uso actual, ya que esto permite el cálculo de la rentabilidad actual y futura en cada una de las zonas en consideración. Para tales fines, se consultaron varios trabajos de zonificación, empezando por el de Guagliardo (1981) a quien se debe un mapa ecológico de la Provincia de Buenos Aires, demarcado en base a los siguientes criterios: regimen de precipitaciones, análisis de suelos, la actividad principal y secundaria de cada área, y por ultimo, precios por hectárea en dólares. El área en estudio está individualizada como región agrícola sur, que se subdivide a su vez en nueve subregiones de acuerdo a los mismos criterios. El mapa de Guagliardo es, casi coincidente con el "Estrato trigo, zona III", elaborado por el CONADE-CFI (1964) para la provincia de Buenos Aires, y que es muy utilizado en los trabajos empíricos tanto del I.N.T.A. como de la 


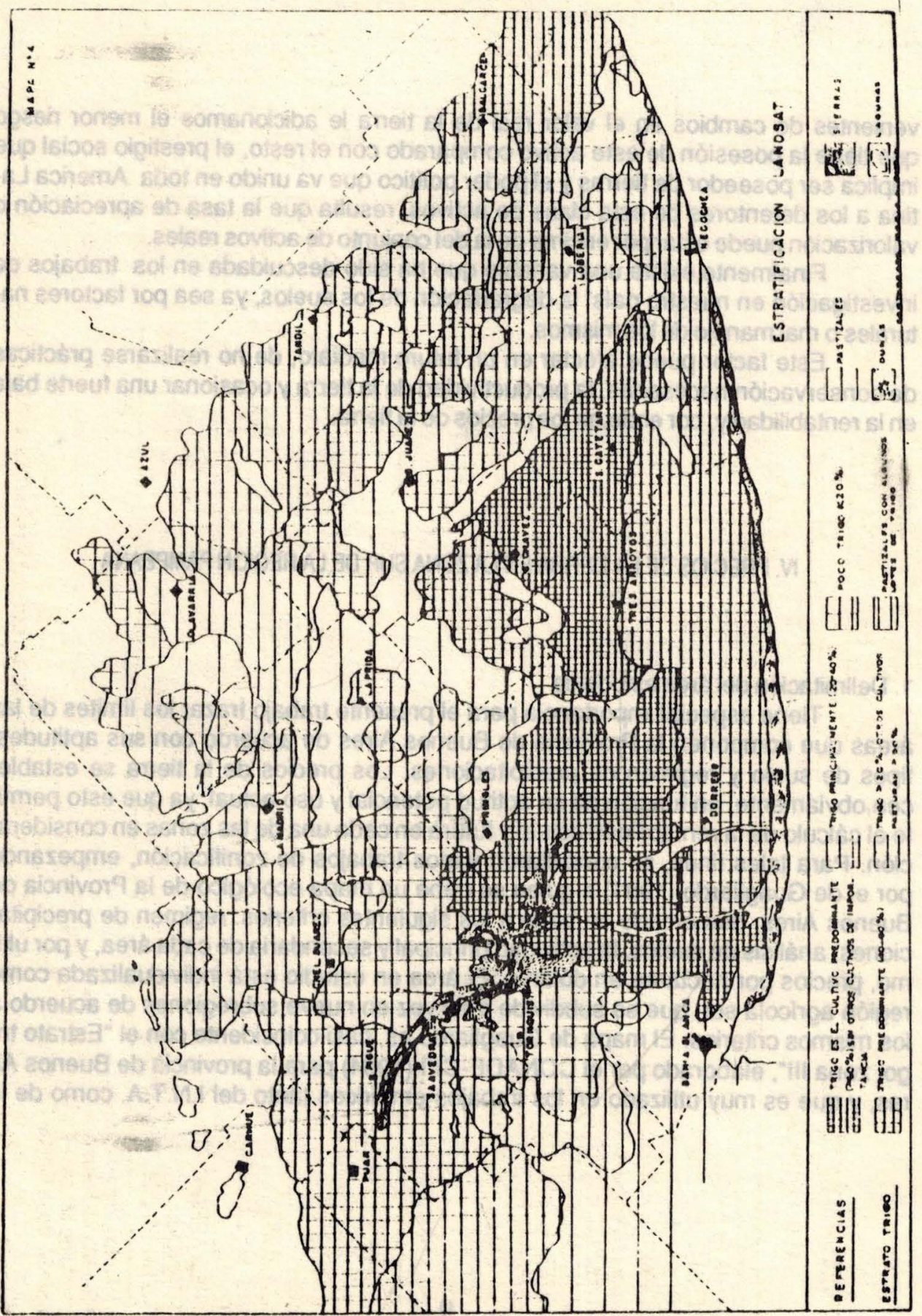


Cuadro № 1

POBLACION DE LA ZONA SUR PAMPEANA ENTRE 1960 Y 1980

\begin{tabular}{|c|c|c|c|c|c|c|c|c|c|c|c|}
\hline \multirow{3}{*}{$\begin{array}{c}\text { PARTIDOS DE LA ZONA } \\
\text { SUR PAMPEANA }\end{array}$} & \multirow{3}{*}{$\begin{array}{l}\text { SUP. } \\
\text { en } \mathrm{Km}^{2}\end{array}$} & \multicolumn{4}{|c|}{ CENSO 1960} & \multicolumn{6}{|c|}{ CENSO 1980} \\
\hline & & POBLAC. & $\mathrm{Hab} /$ & Urbana & Rural & POBLAC. & Hab/ & Urbana & Rural & & $\%$ \\
\hline & & TOTAL & $\mathrm{Km}^{2}$ & & & TOTAL & $\mathrm{Km}^{2}$ & & & Urb. & Rural \\
\hline RAICARCF & 1.120 & 37.477 & 9,1 & 20.540 & 16.937 & 39.544 & 9,6 & 29.406 & 10.138 & 74,4 & 25,6 \\
\hline BAHI & 2.300 & 153.631 & 66,8 & 132.437 & 21.194 & 234.047 & 101,8 & 228.273 & 5.744 & 97,5 & 2,5 \\
\hline BENITO & 5.285 & 17.303 & 3,3 & 9.535 & 7.768 & 20.847 & 3,9 & 14.968 & 5.879 & 71,8 & 28,2 \\
\hline CORONEL DORREGO & 6.020 & $21.147^{1}$ & 3,5 & 11.511 & 9.636 & $18.667^{2}$ & 3,2 & 12.852 & 5.815 & 68,8 & 31,2 \\
\hline CORONEL PRINGLES & 5.245 & 23.048 & 4,4 & 14.180 & 8.868 & 22.068 & 4,2 & 17.033 & 5.035 & 77.2 & 22,8 \\
\hline CNEL, MARINAL, ROSALES & 1.340 & 43.752 & 32,7 & 39.047 & 4.705 & 59.858 & 44,7 & 56.620 & 3.238 & 94.6 & 5,4 \\
\hline CORONEL SUA & 5.985 & 30.211 & 5.0 & 12.900 & 17.311 & 32.354 & 5,4 & 21.130 & 11.224 & 65,3 & 34.7 \\
\hline GENERAL ALVARADO & 1.680 & 19.015 & 11,3 & 11.823 & 7.187 & 25.643 & 15,3 & 21.177 & 4.466 & 82,6 & 17.4 \\
\hline GENERAL LAM & 4.800 & 11.527 & 2.4 & 5.161 & 6.366 & 10.593 & 2,2 & 5.153 & 5.440 & 48,6 & 51,4 \\
\hline GENERAL PUEYRREDON & 1.460 & 224.824 & 154,0 & 213.608 & 11.216 & 434.160 & 297,4 & 419.369 & 14.791 & 96.6 & 3,4 \\
\hline GONZALEZ CHAVES & 3.780 & 12.799 & 3,4 & 5.744 & 7.025 & 12.808 & 3,4 & 8.585 & 4.223 & 67,0 & 33,0 \\
\hline LAPRIDA & 3.440 & 8.721 & 2,5 & 5.816 & 2.905 & 9.066 & 2,6 & 6.553 & 2.513 & 72,37 & 27.7 \\
\hline LOBERIA & 4.765 & $27.671^{3}$ & 5,8 & 16.044 & 11.627 & $17.475^{4}$ & 4.0 & 9.624 & 7.851 & 55,1 & 44.9 \\
\hline MUNICIPIC & 226 & - & - & - & - & 25.652 & 113,5 & 20.873 & 4.779 & 81,4 & 18,6 \\
\hline MUNICIP & 63 & - & E & - & - & 5.326 & 84,5 & 4.459 & 867 & 83,7 & 6,3 \\
\hline M. URB. DE M. HERMOSO & 209 & $\overline{-}$ & $\overline{-}$ & $\overline{-}$ & - & 3.122 & 14,9 & 2.886 & 236 & 92.4 & 7.6 \\
\hline MUN. DE VILLA GESEI & 285 & $\bar{E}$ & 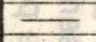 & $\underline{E}$ & $\bar{E}$ & 11.632 & 40,8 & 11.632 & & 100,0 & \\
\hline NECOCHEA & 4.465 & 43.500 & 9,7 & 32.645 & 10.855 & 73.634 & 15,4 & 65.465 & 8.169 & 88,9 & 11.1 \\
\hline PUAN & 6.385 & 20.194 & 3.2 & 11.060 & & 18.485 & 2,9 & 9.408 & 9.077 & 50,9 & 49,1 \\
\hline SAAVEDRA & 3.500 & 16.956 & 4,8 & 10.049 & 6.907 & 18.312 & 5.2 & 13.239 & 5.073 & 72,3 & 27.7 \\
\hline SAN CAYETANO & 3.014 & 10.727 & 3,6 & 5.733 & 4.994 & 8.865 & 3,0 & 5.981 & 2.884 & 67.5 & 32,5 \\
\hline TANDIL & 4.935 & 69.005 & 14.0 & 48.726 & 20.279 & 91.873 & 18,6 & 81.494 & 10.379 & 88,7 & 11,3 \\
\hline TRES ARROYOS & 5.871 & 50.670 & 8,6 & 39.136 & 11.534 & 54.329 & 9,3 & 43.418 & 10.911 & 79,9 & 20.1 \\
\hline TORNQUIST & 4.183 & 9.535 & 2,3 & 3.062 & 6.473 & 10.062 & 2,4 & 4.701 & 5.361 & 46.7 & 53.5 \\
\hline TOTALES ZOI & 82.573 & 851.713 & 10,3 & 648.762 & 202.921 & 1.258 .422 & 15,2 & 1.114 .299 & 44.093 & 88,5 & 11,5 \\
\hline
\end{tabular}

1 Inluído Monte Hermoso

2 Sin Monte Hermoso

3 Incluído M. de la Costa, Pinamar y Villa Gesell

4 Sin M. de la Costa, Pinamar y Villa Gesell

Euente: Censos Nacionales de Población correspondientes a los años 1960 y 1980. 
Secretaria de Agricultura, Ganadería y Pesca de la Nación. Sin embargo, en la actualidad, este ultimo organismo, con el objeto de alcanzar una mayor homogenización de las áreas bajo estudio, está en vías de modificar su sistema de zonificación siguiendo metodos más modernos, como son las imágenes satelitarias.

Se reproduce en el mapa Nro 1 la "estratificación Landsat" en lo que se refiere al estrato trigo, que refleja un estudio minucioso de la región triguera, subdividida de acuerdo al procentaje de hectáreas dedicadas al cultivo de trigo y de otros cereales invernales. En base a esta información se definió el área objeto de estudio. En el Cuadro I se enumeran el conjunto de partidos que la componen, así como la evolución de la población rural y urbana entre 1960 y 1980.

\section{Tendencias en el valor real de la tierra.}

Para elaborar una serie de precios de la tierra que tuviera un nivel significativo de confiabilidad se tomaron en consideración los valores obtenidos en remates publicos (comerciales y judiciales); los datos recogidos por informadores autorizados de la Secretaría de Agricultura, Ganadería y Pesca de la Nación; valores estimativos de la revista "La Chacra", valores fiscales de la Dirección General Impositiva y precios fijados por las inmobiliarias dedicadas a la venta de campos ( $\mathrm{Pe}$ dro y Antonio Lanusse S. A., Agro-Palermo S. A., Nordheimer y Minola, etc.). Se confrontaron y analizaron las series asi obtenidas, se eliminaron fracciones menores a 100 hectáreas y las que por su proximidad a centros urbanos pudieran ser destinadas a fines no agropecuarios.Se eligieron, preferentemente, predios de similar tamaño y nivel de mejoras. Finalmente, se calcularon los valores medios representativos de cada afio y se deflactaron por el índice de precios mayoristas no agropecuarios total (base $1970=100$, índice de conversión $=5,641$ ), lo cual permitió confeccionar una serie de valores de la tierra constantes. Tal como se puede observar en el Cuadro II y la Fig. 1 los precios de la tierra en la zona sur pampeana durante el periodo considerado muestran un proceso casi contínuo de suba, interrumpido en algunos años por fuertes bajas y aumentos debidos a causas coyunturales, pero que de ningun modo afectan la tendencia general. Nuestra serie arranca con una caída que se incia en 1.951 y continúa hasta 1.954 , debido a la política 
Cuadro 2

$$
\begin{gathered}
\text { EVOLUCION DE LOS PRECIOS DE LA TIERRA EN LA ZONA } \\
\text { SUR PAMPEANA A VALORES CONSTANTES }
\end{gathered}
$$

\begin{tabular}{lc||cc}
\hline AÑO & $\begin{array}{c}\text { PRECIOS DE LA TIERRA } \\
\text { (\$LEY 1970/HA) }\end{array}$ & AÑO & $\begin{array}{c}\text { PRECIOS DELA TIERRA } \\
\text { (\$LEY 1970/HA) }\end{array}$ \\
\hline & & & \\
1950 & 428,68 & 1968 & 573,20 \\
1951 & 310,89 & 1969 & 555,68 \\
1952 & 283,69 & 1970 & 530,00 \\
1953 & 280,94 & 1971 & 711,30 \\
1954 & 270,97 & 1972 & 943,01 \\
1955 & 374,27 & 1973 & 848,03 \\
1956 & 315,66 & 1974 & $1.185,35$ \\
1957 & 345,52 & 1975 & $1.306,00$ \\
1958 & 485,78 & 1976 & $1.887,43$ \\
1959 & 192,02 & 1977 & $1.565,15$ \\
1960 & 240,33 & 1978 & 821,67 \\
1961 & 557,43 & 1979 & 807,18 \\
1962 & 436,48 & 1980 & 941,96 \\
1963 & 813,33 & 1981 & 640,82 \\
1964 & 680,71 & 1982 & 815,43 \\
1965 & 537,18 & 1983 & 978,63 \\
1966 & 538,31 & 1984 & 983,68 \\
1967 & 566,69 & 1985 & 831,74 \\
\hline
\end{tabular}

Fuente: Elaboración propia. 


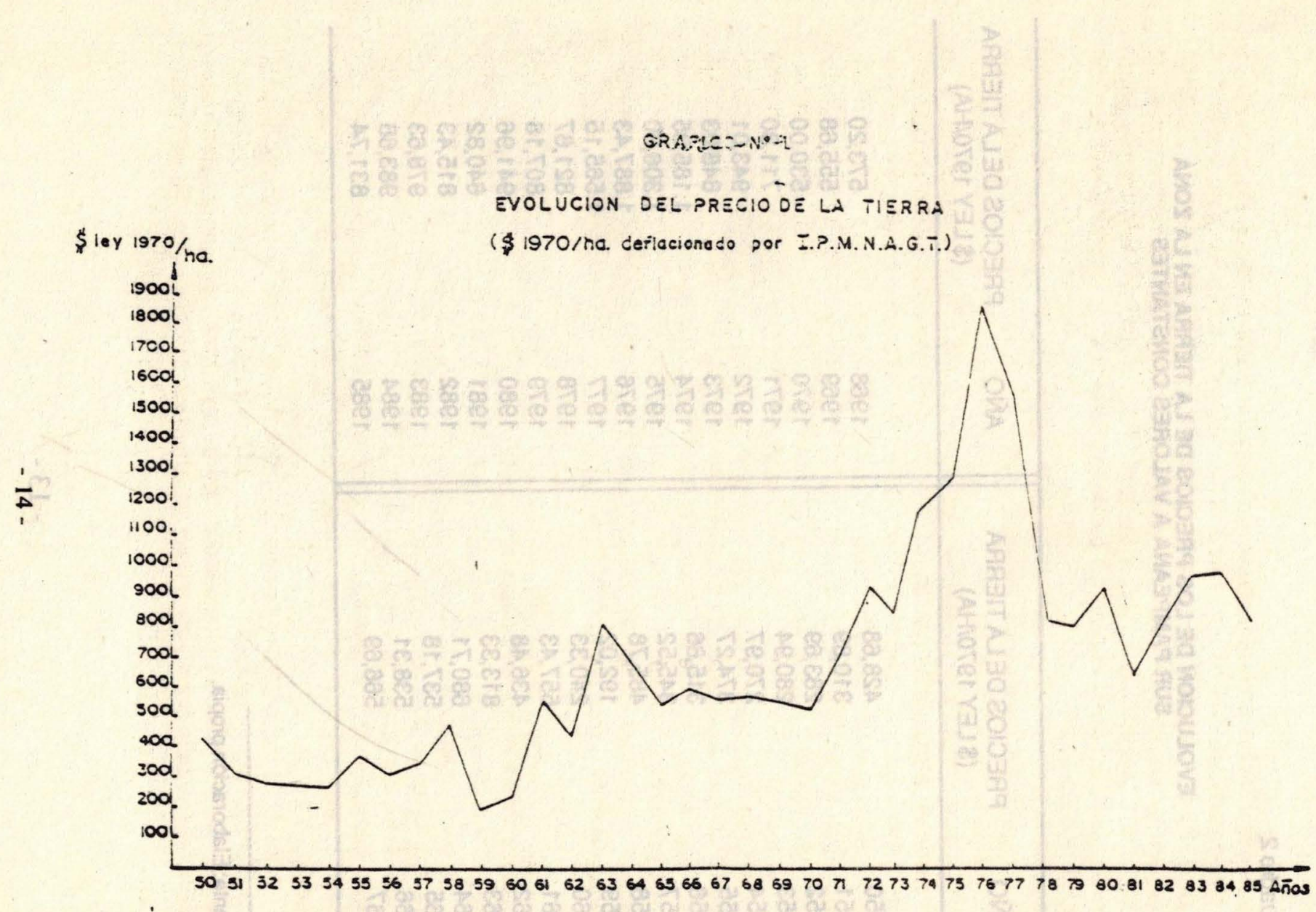


imperante que dirige preferentemente su atención hacia el desarrollo industrial y los problemas redistributivos y no hacia el crecimientos del sector agropecuario. A partir de 1959 en adelante, hay una recuperación notable de los valores, fenómeno que parece relacionarse con los aumentos de productividad registrados en la decada del 60 y la política favorable al sector (credito subsidiado, exenciones impositivas) que favorecieron la demanda de tierras rurales. Los valores se estabilizan en la segunda mitad del periodo. Comienza una carrera alcista a principio de 1971 que llega a picos elevadísimos de 1974 a 1977. Es necesario resaltar la notable incidencia que pudieron haber tenido en estos años los incrementos significativos de los precios de los principales granos en el mercado mundial, como asimismo el llamado "bull market"manifestado a mediados del 70, por la acción compradora de un nuevo grupo de adquirentes nacionales y extranjeros ajenos al sector agropecuario, que, habiendo acumulado fuertes capitales, necesitaban a los efectos impositivos canalizarlos fuera de sus actividades normales. El descenso que ya se manifestaba a comienzos de 1977 , se profundiza en 1978 y 1979 y alcanza niveles similares a los registrados a comienzos de la decada del setenta, superiores, sin embargo, al promedio de los vigentes en la del sesenta. Entre otros factores, este hecho puede deberse tambien a la baja que se manifiesta en el precio de los granos a nivel mundial a fines de la decada en consideración y que continua con niveles relativamente deprimidos durante la decada del 80 . Por esta razón, los valores de la tierra en los primeros años del 80 ya habian sido alcanzados en 1972-73, y la fuerte contracción de1981 es debida seguramente a la iliquidez monetaria que se registró en toda clase de activos incluyendo la tierra.

3. Variables correlacionadas con los precios de la tierra.

En la zona sur de la región pampeana, la actividad agrícola predominante es el cultivo del trigo, su área ecológica por excelencia. A partir de 1978/79, se incorpora el girasol como segundo cultivo de importancia; siguen en orden decreciente la cebada cervecera, la avena, el lino, el sorgo granífero y el maiz.La ganaderia por su parte, ha ido perdiendo peso, debido a la caída en terminos reales del precio de la carne a nivel interno y a la dificultad de su colocación en los mercados externos.

A los fines del presente trabajo, se tomaron los ingresos brutos derivados de los cultivos de trigo y girasol, y en el caso de la ganadería, los precios del novillo, ya que en materia de rendimientos de la producción ganadera no existen datos agregados (sólo datos puntuales a nivel de empresa agropecuaria). Se estimaron 
los impuestos pagados por el sector agropecuario desde1956 a 1980; los saldos de prestamos bancarios destinados al sector primario y total de la economía; incluso se calculó la relación porcentual que estos representan sobre el total de prestamos; se tomó en consideración la evolución de las tasas de interes deflacionadas por distintos índices de precios; el tipo de cambio comprador Banco Nación para exportaciones tradicionales (pesos por dólar) y el tipo de cambio efectivo para trigo y girasol. Finalmente, se analizó la evolución de los precios de los insumos agropecuarios relativos al novillo, al girasol y al trigo que comienzan a elevarse en forma continuada recien a partir de1974-75.

En todos los casos se construyeron cuadros y gráficos, a fin de visualizar las tendencias de las variables mencionadas y los hechos susceptibles de explicar sus fluctuaciones de corto plazo.

\section{Modelo econometrico y sus resultados.}

Con el objeto de separar los efectos de las variables a las que se asigna importancia en la determinación de los precios de la tierra en la zona estudiada, se ha recurrido a un modelo econométrico de regresión múltiple, calculado por mínimos cuadrados ordinarios. En todos los casos, los ajustes fueron hechos a traves de funciones lineales.

El numero máximo de variables explicativas que se consideraron en cada ecuación es de seis. El período de tiempo más extenso (limitado muchas veces por la información básica) abarca de 1950 a 1985. Se recurrió a una dummy (1) que en un caso se limita a los anos 1974-77, ya que, como se evidencia en los datos de las series de precios de la tierra obtenidos, responden a guarismos que superan ampliamente los valores normales medios. Una segunda dummy (2) utilizada abarca los ańos 1974-76 a los fines de tener en cuenta el tiempo correspondiente al período presidencial peronista, yà que en 1977, la política sectorial parece representar el inicio de una nueva orientación. 
Cuadro № 3

COEFICIENTES OBTENIDOS EN BASE A LAS ESTIMACIONES REALIZADAS POR MINIMOS CUADRADOS

\begin{tabular}{|c|c|c|c|c|c|c|c|c|c|c|c|c|c|c|c|c|c|c|}
\hline \multirow{2}{*}{ ECUACIONES } & \multicolumn{2}{|c|}{$\begin{array}{l}\text { Ing. Bruto } \\
\text { Trine }\end{array}$} & \multicolumn{2}{|c|}{$\begin{array}{l}\text { Ing. Bruto } \\
\text { Girnsol }\end{array}$} & \multirow{2}{*}{$\begin{array}{l}\text { Precio } \\
\text { Novillo }\end{array}$} & \multirow{2}{*}{$\begin{array}{c}\text { Impuesto } \\
\text { Nacional } \\
\text { Aprop. }\end{array}$} & \multicolumn{2}{|c|}{$\begin{array}{l}\text { Tasa de interes } \\
\text { Activa Relativa }\end{array}$} & \multirow{2}{*}{$\begin{array}{l}\text { Tipo cambio } \\
\text { comprador } \\
\text { o. Export } \\
\text { tradic. }\end{array}$} & \multirow{2}{*}{$\begin{array}{l}\text { Tasa de } \\
\text { cambio } \\
\text { inflac. } \\
\text { (l.P.C.) } \\
\end{array}$} & \multicolumn{2}{|c|}{$\begin{array}{c}\text { Tipo de cambio } \\
\text { efectivo }\end{array}$} & \multicolumn{2}{|c|}{ DUMMY } & \multirow{2}{*}{$R_{3}$} & \multirow{2}{*}{$\begin{array}{c}R 2 \\
\text { corrogido }\end{array}$} & \multirow{2}{*}{$\begin{array}{c}\text { Error } \\
\text { Standard }\end{array}$} & \multirow[t]{2}{*}{$D \cdot W$} \\
\hline & 1 & 1 & t & $t-1$ & & & $\frac{\text { ACt }}{\text { AI! }}$ & 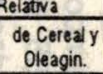 & & & $\frac{\mathrm{e}}{\text { Trigo }}$ & $\frac{\text { ctivo }}{\text { Girasol }}$ & $\begin{array}{l}1 \\
1974^{\circ} \\
1977\end{array}$ & $\begin{array}{l}2 \\
1974 \\
1976\end{array}$ & & & & \\
\hline $\begin{array}{l}1 \\
1956-1980 \\
\end{array}$ & $\begin{array}{r}2,60 \\
(1,95) \\
\end{array}$ & 5 & $\begin{array}{l}0,0556 \\
0,0410 \\
\end{array}$ & & $\begin{array}{r}169 \\
(0,511) \\
\end{array}$ & $\begin{array}{l}0,442 \\
(2,20) \\
\end{array}$ & 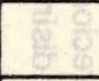 & $\begin{array}{r}2,60 \\
(0,808) \\
\end{array}$ & & & Ez & 7 & & $\begin{array}{r}485 \\
(1,90) \\
\end{array}$ & 0,66 & 0,548 & 271,19 & 1,486 \\
\hline $\begin{array}{l}\text { I } \\
1960-1980\end{array}$ & $\begin{array}{r}1,65 \\
(1,79)\end{array}$ & 4 & $\begin{array}{l}2,56 \\
(2,23)\end{array}$ & 8 & $\begin{array}{r}-2,82 \\
(1,14)\end{array}$ & $\begin{array}{l}0,146 \\
(0,936)\end{array}$ & 50 & $\begin{array}{r}-8,26 \\
(3,3)\end{array}$ & $\begin{array}{l}-170 \\
(3,81)\end{array}$ & & 6 & & & & 0,836 & 0,766 & 189,83 & 1,937 \\
\hline 1950-1985 & & $\begin{array}{l}1,38 \\
(1,57)\end{array}$ & 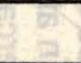 & $\begin{array}{l}1,68 \\
(2,51)\end{array}$ & & 5 & $\begin{array}{l}-3,89 \\
(-1,81)\end{array}$ & 8 & 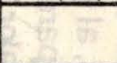 & $\overline{5}$ & $\begin{array}{c}104 \\
(1,06)\end{array}$ & 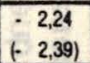 & & $\begin{array}{r}482 \\
(2,86)\end{array}$ & 0,73 & 0,674 & 217,18 & 1,751 \\
\hline $\begin{array}{l}N \\
1950-1985\end{array}$ & $\begin{array}{l}2,19 \\
(2,92)\end{array}$ & $x^{2}$ & $\begin{array}{c}1,56 \\
(2,85)\end{array}$ & 5 & & 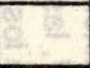 & $\begin{array}{r}-3,68 \\
-1,95)\end{array}$ & $a^{2}$ & 남 & 8 & $\begin{array}{c}26,7 \\
(0,317)\end{array}$ & $\begin{array}{r}159 \\
(-1,99)\end{array}$ & & $\begin{array}{r}403 \\
(2,71) \\
\end{array}$ & 0,79 & 0,747 & 189,75 & 1,843 \\
\hline $\begin{array}{l}V \\
1950-1985\end{array}$ & $\begin{array}{l}2,04 \\
(3,04)\end{array}$ & 2या & $\begin{array}{l}1,21 \\
(2,46)\end{array}$ & $=$ & $\sqrt{9}$ & E & $\begin{array}{l}-2,05 \\
(-1,12)\end{array}$ & 0 & G & & $\begin{array}{r}12,9 \\
(0,172)\end{array}$ & $\begin{array}{r}-132 \\
-1,83\end{array}$ & $\begin{array}{c}506 \\
(3,94)\end{array}$ & & 0,829 & 0,793 & 171,49 & 1,944 \\
\hline $\begin{array}{l}\text { U } \\
1950-1985\end{array}$ & $\begin{array}{l}2,01 \\
(2,87)\end{array}$ & 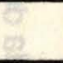 & $\begin{array}{l}0,185 \\
(0,314)\end{array}$ & a & 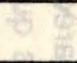 & & 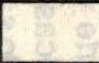 & 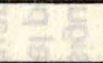 & 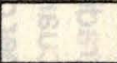 & $\begin{array}{l}73,7 \\
(3,34)\end{array}$ & $x$ & & $\begin{array}{c}686 \\
(6,52)\end{array}$ & 3 & 0,794 & 0,767 & 182,14 & 1,105 \\
\hline $\begin{array}{l}V 17 \\
1970-1985\end{array}$ & $\begin{array}{l}3,52 \\
(2,39)\end{array}$ & 9 & $\begin{array}{l}0,206 \\
(0,206)\end{array}$ & 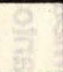 & 7 & & $\begin{array}{l}-9,02 \\
(-4,22)\end{array}$ & 38 & 89 & $\begin{array}{r}25,8 \\
(0,872)\end{array}$ & the & & & & 0,765 & 0,679 & 197,45 & 2,340 \\
\hline
\end{tabular}

NOTA: Los valores entre paréntesis debajo de cada coeficiente de la regresión son los I student hallados. 
Los resultados alcanzados se vuelcan en el Cuadro 3. Los valores del $R^{2}$ de todas las ecuaciones oscilan entre 0,70 y 0,85 , salvo en la ecuación I. Los estadísticos de Durbin-Watson correspondientes adquieren valores cercanos a 2 , excepto en la ecuación VI, lo cual torna significativos algunos resultados.

Surge del análisis de la ecuación II -que comprende el período 1960/80que los ingresos derivados de trigo y girasol ofrecen un poder explicativo satisfactorio, al igual que la tasa de interes deflacionada por un índice relativo a cereales y oleaginosos. Las elasticidades parciales para los ingresos provenientes del trigo y del girasol con respecto a los precios de la tierra son de 0,52 y 0,76 respectivamente, lo que, si bien no redunda en cambios espectaculares en el valor de la tierra, hace que un incremento del $10 \%$ en los cereales nombrados tienda a producir un aumento del 5 al 7,6\% en el precio de la tierra. Por otra parte, el signo negativo de la variable tasa de interés pone de relieve que ante alzas en la tasa de interés, los inversores dirigen sus miradas hacia activos más redituables, lo cual produce una baja en la demanda de tierras rurales, por ende en sus precios. A la inversa, un descenso de dicha tasa favorece las inversiones en el sector rural, ya que, por un lado, la tierra renta más en terminos relativos, y, por otro, ofrece la posibilidad de efectuar mejoras a menor costo crediticio. En cambio, el tipo de cambio comprador Banco Nación para exportaciones tradicionales, al igual que los precios del novillo y los impuestos nacionales agropecuarios no aparecen con los signos esperados teóricamente, pese a que, en el primer caso, el valor " $t$ " Student es suficientemente alto.

En las ecuaciones siguientes se amplía el tiempo a fin de tener en cuenta todo el período que abarca el presente trabajo:1950-85. No se perderá de vista las dificultades que aparecen cuando se analizan periodos tan extensos, sobre todo en países sujetos a una fuerte variabilidad de precios internos y externos, y donde se aplican políticas económicas sectoriales muy disímiles.Sin embargo, los resultados estadisticos siguen siendo satisfactorios.

En las ecuaciones III, IV y V, las variabtes seleccionadas son análogas. La única diferencia reside en el caso de los ingresos brutos provenientes de la producción de girasol y trigoque aparecen desfasados en un afio en la ecuación III. Considerando los rezagos lógicos que se presentan via precios en este tipo de actividad, no se abtuvo sin embargo, mejoras en los resultados. A nuestro juicio, tal circunstancia constituye tanto el resultado del adelanto tecnológico, que permite levantar mas de una cosecha en el curso de un af́o, como del perfeccionamiento de los medios de comunicación, que facilitan al productor agropecuario estar informado al instante sobre el estado de los mercados cerealeros nacionales tanto como extranjeros.

Se han incorporado la dummy (2) en las ecuaciones III y IV, y la dummy (1) 
en la V. De acuerdo al "t" Student alcanzado, esta ultima ofrece un mayor poder explicativo. Se ha introducido en el análisis de estas ecuaciones la variable tipo de cambio efectivo para trigo y girasol, con la esperanza de recoger de este modo con más exactitud las variaciones en el poder adquisitivo sectorial.Sin embargo, la presencia de esta variable en la misma ecuación ha originado un problema de colinealidad, que le ha quitado poder de significación y signo no previsto de acuerdo al esquema teórico. Por lo tanto, se hace desestimable esta variable en la regresión.

En las ecuaciones VI y VII se ha introducido una variable supuestamente de notoria influencia y de gran fuerza explicativa, segun el análisis teórico realizado: la inflación. A tal fin, recurrimos a una tasa inflacionaria de cambio que refleja los cambios en la tasa de crecimiento de los precios en el período considerado. La ecuación VI recoge el significado de esta variable que, junto con la dummy (1) y los ingresos provenientes del trigo, ofrecen explicaciones del precio de la tierra en casi un $80 \%$. La elasticidad de la tasa inflacionaria de cambio señala que un incremento de $10 \%$ en la tasa anual de aumentos de precios produce incrementos del1,16\% en los precios de la tierra. Este resultado, que carece aparentemente de significación puede tener importancia en un país con persistentes procesosinflacionarios como Argentina, donde el hecho saliente lo da el cambio en el ritmo inflacionario más que la inflación por si misma. En la ecuación VII se toma un período más breve (1970/85), sobre todo para verificar lo ya señalado anteriormente, que los resultadosdeben mejorar sensiblemente ya que los datos cubren un periodo más homogéneo en cuanto al proceso inflacionario que si bien es persistente en Argentina se ha agravado en la última década y media.

El coeficiente de elasticidad de los ingresos provenientes del trigo pasan de 0,52 a 0,93 , lo que significa que el $10 \%$ de variación en trigo produce casi el mismo efecto $(9,3 \%)$ en la variación de los valores de la tierra, lo que denota la gran repercusión que en materia de rendimientos ha experimentado el trigo/ha en los ultimos anos.

Por otro lado, la tasa de interés con una elasticidad igual a 0,13 en la última década y media, produce un incremento del 1,3\% en los valores de la tierra y juega por lo tanto, un rol importante en los períodos más breves. Por otra parte, la tasa inflacionaria de cambio pierde poder explicativo frente a la tasa de interés, siendo una razón posible que la primera ha pasado a constituir una variable marco de referencia frente a la segunda que responde a una politica económica de corto plazo, de indole coyuntural y de efectos mayores. Los resultados alcanzados son un $\mathrm{R}^{2}$ de 0,765 y un D-W de 2,34, valores ambos que dan una idea de un buen ajuste.

Se efectuaron otras pruebas empíricas con variables no tenidas en cuenta en el análisis precedente, tales como prestamos bancarios en cartera viva con desti- 
no al sector primario y la inclusión de una dummy que correspondió a una periodización realizada con el siguiente criterio: otorgarle valor 0 a los añosde política económica desfavorable al sector agropecuario y valor 1 al caso contrario; en ambos casos los resultados no fueron losesperados. En cuanto a tos insumos agropecuarios, también sucedió lo mismo, tal vez como consecuencia de haber tomado un período breve de datos 1974/85 y demasiadas variables explicativas (con lo que se trabaja con muy pocos grados de libertad, lo cual produce una disimilitud manifiesta entre el $R^{2}$ corregido y el $R^{2}$ ).

\section{CONCLUSION}

La cuestión fundamental planteada en este trabajo gira en torno al siguiente interrogante: ¿los precios de la tierra sedeterminan teniendo en cuenta aspectos de orden puramenteproductivo o tal vez intervienen en su formación, en especial en la ultima década y media, otros factores que operan desde fuera del sector agropecuario?.Los resultados alcanzados indican que más del $50 \%$ del valorde las tierras rurales vienen relacionados con los ingresos de las actividades directamente vinculadas al factor tierra y que constituyen los cultivos básicos de la zona sur pampeana. El trigo y el girasol en las regresiones planteadas confirman su incidencia; en el primer caso, con un coeficiente de elasticidad de 0,93 para el período 1970/85 que marca una casi simetria entre variaciones porcentuales en los ingresos del trigo y el precio de la tierra. Esto tiene implicancias en las políticas económicas que dirigidas al sector agropecuario afectan de algun modo el precio de los cereales, ya sea en forma directa (precio sostén) o indirecta (retenciones, tipo de cambio), o incentivan el avance tecnológico, pues ambos elementos, precios y rendimientos, son de indudable repercusión sobre los precios de la tierra.

Pero tal vez uno de los resultados más interesantes del análisis consiste en comprobar la importancia de las variables de indole monetaria y especulativa en la determinación de los precios de la tierra.

En la parte II del presente trabajo se mencionan estudios que tienen en cuenta este tipo de variables a fin de detectar unaexplicación plausible del alejamiento que muy frecuentemente se da entre los precios de la tierra y los de los productos agropecuarios. Sería propicio en estas circunstancias volver a recordar la cuidadosa definición que diera David Ricardo del concepto de renta de la tierra, pues la posibilidad de considerar al suelo no ya como un soporte de la actividad productiva, sino como un activo de cartera sumamente preciado para aquellos que buscan oportunidades para sus capitales, crea un contexto nuevo de análisis.

La tasa de interes y la tasa inflacionaria de cambio han servido a los fines ex- 
puestos; en nuestro modelo econometrico la primera tiene un rol importante como reguladora de la oferta y la demanda de activos, en países subdesarroladas, y la segunda es una variable de gran utilidad para el análisis de largo plazo, pues registra en forma clara el fenómeno de aumento de los precios de la tierra en terminos de su apreciación como "reserva de valor" frente al deterioro adquisitivo de la moneda. Este resultado que consideramos de importancia ratifica la aparición de un conceptode renta de origen distinto a los planteados en la parte I de nuestro estudio donde efectuamos una rápida revisión de las teorias existentes en el pensamiento económico. Esta renta que denominamos "renta especulativa", queda confirmada, a traves de la tendencia alcista de los precios de la tierra en la zona sur pampeana que resultó correlacionada con los cambios experimetados en los niveles de precios de la economía. A nuestro entender, políticas económicas encaminadas a aumentar la productividad del sector agropecuario no deben dejar de lado las consideraciones anteriores. Al verificarse la presencia de una "renta especulativa" 6 comprende que haya existido una tendencia al mantenimiento de explotaciones improductivas o con bajos rendimientos, ya que el origen de la compra no estuvo inspirado sólo en motivos de orden productivo sino tambien en razones de índole financiera, asociado a aspectos coyunturales.

Finalmente, queremos destacar posibilidades ciertamente trascendentes en el comportamiento de la variable "degradación del suelo" susceptible de producir efectos económicos negativos en un futuro cercano, en virtud del gran avance de los procesos erosivos acompañados de una agricultura intensiva y de una falta casi total de prácticas conservacionistas. Tema este, que abre interrogantes sin resolver que serian utiles en lo que hace al impacto que provocaria sobre las variables económicas y su consecuencia para el desarrollo agrario 


\section{REFERENCIAS}

BARRAGLOUGH, E.(1966). "Estructura Agraria en siete paísesLatinoamericanos". Economía de la tierra, Noviembre.

BLAUGH, M. ( 1968). La teoría económica actual. Barcelona: Luis Miracle.

BUCHANAN, D. H.(1929) "The Historical Approach to Rent and Price Theory". Económica, 26, junio.

BULLOCK, J. B., W. L. NIENWONDT y E. C. PASSOUR Jr. (1977) "Lands Values and Allotment Rents", Agricultural Economics, vol. 59, Mayo.

CARACCIOLO DE BASCO, M. y NEIMAN G. (1979) Evaluación socio-económica de las explotaciones agropecuarias constituídas en la Provincia deBuenos Aires en el período Abril 1976 a Diciembre 1977. ServicioNacional de Economía y Sociología rural, Publicación 119, Buenos Aires.

CARR, J. y SMITH L. (1975)"Public Land Banking and the Prices of Land", Land Economics, II, 4, noviembre.

CHASE, L. (1980)" Inflation, Capital Gains and Farmers Economics Well Being". Canadian Journal of Agricultural Economics, agosto .

CIRIACY-WANTRUP, S. V.( 1960). Conceptual Problems in Projecting the Demand for Land and Water. Chicago: University of Illinois Press."The New Competition for Land and Some Implications.for Public Policy". Natural Ressources Review, California, octubre.

CONADE-CFI. (1964). Tenencia de la tierra. Aspectos de estructura agraria y su incidencia en el desarrollo agrario argentino. Buenos Aires.

FERNANDEZ, O.(1985). Tierras áridas y semiáridas: dos tercios del territorio. CRIBABB. (Centro Regional de Investigaciones Básicas y Aplicadas, Bahía Blanca): Marzo.

FLICHMAN, G.(1977). La renta del suelo y el desarrollo agrario argentino. Mexicn: Siglo XXI. (1978) Notas sobre el desarrollo agropecuario en la Región Pampeana Argentina (o por que Pergamino no es lowa?). Buenos Aires: Estudios Cedes, Vol. 1, 4-5.

GARCIA LABOUGLE, J.(1983) "El valor de la tierra". Buenos Aires: Diario La Nación, enero.

GRAY, E.P.B.(1982)."Agricultural Land Prices. The Effect of the Terms of Sale". Canadian Journal of Agricultural Economics, Junio-Julio .

HARRIS, D. G.(1977). "Inflation Indexed Supports and Land Values". Journal of Agricultural Economics, 59, 3, agosto .

HEADY, E. V. y TWEETEN, L. G.(1963). Resource Demand and Structure of the Agricultural Industry, lowa State Press. 
HOOVER, D. M.(1960). A Study of Agricultural Land Prices in the United States 1911-58. Disertación no publicada, Ph. D., University of Chicago. LANUSSE P. y A. (1967). Evolución del precio de la tierra para el Partido de Rauch entre 1936-66. Buenos Aires, abril .

LEE, W. y RASK, N.(1986)."Inflation and Crop Profitability: How Much Land Farmers Pay for Land?", American Journal of Agricultural Economics, 58, 5, diciembre.

MARTINEZ, J. C., PINEIRO M. y CHEVALIER C.(1976). "Nuevamente en torno al problema de asignación de recursos en el sector agropecuario pampeano. Una nota complementaria a la polemica Flichman-Braun", Desarrollo económico, 61.

MARX, K.(1959). El Capital. Mex̧ico: Fondo de Cultura Económica.

PASSOUR, E. D. Jr.(1973). "Real Property Taxes and Farm Real Estate". American Journal of Agricultural Economics, 55, 4, noviembre.

PINEIRO, M.(1970)."El impuesto a la tierra, su impacto potencial sobre la producción agropecuaria", Económica, 3,setiembre-diciembre .

RAS, N.(1977). El desarrollo agropecuario de la Argentina. Buenos Aires: Hemisferio Sur.

RAS, N. y LEVIS R.(1978). El precio de la tierra, su evolución entre los años 1916-78. Buenos Aires: Sociedad Rural Argentina.

RECA, L.(1982). El sector agropecuario pampeano, situación actual y posibilidades de crecimiento. Buenos Aires: Fundeco.

RECA, L. y CIRIO F.(1983).Fertilizantes químicos: fuente de crecimiento subutilizada en la agricultura pampeana. Buenos Aires: Fundeco.

REYNOLDS, J. E. y TUNNIONS F.(1969).Factors Affecting Farm Land Values in the United States. lowa University State.

RICARDO, D.(1959). Principios de Economía Política y Tributación. Mexico: Fondo de la Cultura Económica.

RUTTAN, V.(1961)."The Impact of Local Population pressure on Farm Real Estate Values in California". Lands Economics, 37, 1-4.

SCHUH, E. y SCHARLACH W.(1963). Quantitative Analysis of Some Farm and Nonfarm Determinants of Agricultural Land Values. Bulletin Technical 821. Purdue University.

SCHULTZ, T. W.(1951). "La importancia de la declinación de la tierra agrícola", diciembre .

SMITH, A.(1933). Investigaciones de la naturaleza y causas de la riqueza de las naciones. Barcelona: Bosch.

SOJIT, A.(1968). Factors Affecting Lands Values in Argentina Research Essay. Berkeley: University of California. 
VAN VUUREN.(1975). "Land Valuation for Tax Assessment and Land Use Planning. The Search for and Use of Appropriate Measures". Canadian Journal of Agricultural Economics, 23, 2, julio. (1968). Agricultural Land Prices and Returns in an Advanced Urban and Industrilized Economy. Disertación no publicada, Ph. D., Berkeley:University of California.

ZEMBORAIN, S.(1973). La verdad sobre la propiedad de la tierra enArgentina. Buenos Aires: Instituto de Estudios Económicos de la Sociedad Rural Argentina. 